\title{
The Healthy Nordic Diet and Mediterranean Diet and Incidence of Disability 10 Years Later in Home-Dwelling Old Adults
}

\section{Perälä, Mia-Maria}

2019-05

Perälä , M-M , von Bonsdorff , M B , Männistö , S , Salonen , M K , Simonen , M , Pohjolainen , P , Kajantie , E , Rantanen , T \& Eriksson , J G 2019 , ' The Healthy Nordic Diet and Mediterranean Diet and Incidence of Disability 10 Years Later in Home-Dwelling Old Adults ', Journal of the American Medical Directors Association , vol. 20 , no. 5 , pp. 511-+ . https://doi.org/10.1016/j.jamda.2018.09.001

http://hdl.handle.net/10138/314396

https://doi.org/10.1016/j.jamda.2018.09.001

cc_by_nc_nd

acceptedVersion

Downloaded from Helda, University of Helsinki institutional repository.

This is an electronic reprint of the original article.

This reprint may differ from the original in pagination and typographic detail.

Please cite the original version. 
1 The healthy Nordic diet and Mediterranean diet and incidence of disability 10 years later in home-dwelling old adults

4 Mia-Maria Perälä $\mathrm{PhD}^{1,2}$, Mikaela B von Bonsdorff $\mathrm{PhD}^{2,3}$, Satu Männistö $\mathrm{PhD}^{1}$, Minna K

5 Salonen $\mathrm{PhD}^{1,2}$, Mika Simonen $\mathrm{PhD}^{4}$, Pertti Pohjolainen $\mathrm{PhD}^{5}$, Eero Kajantie $\mathrm{MD}, \mathrm{PhD}^{1,6,7}$, Taina

6 Rantanen $\mathrm{PhD}^{3}$, Johan G Eriksson $\mathrm{MD}, \mathrm{PhD}^{1,2,8,9}$

$8 \quad{ }^{1}$ Department of Public Health Solutions, National Institute for Health and Welfare, Helsinki,

9 Finland

$10 \quad{ }^{2}$ Folkhälsan Research Center, Helsinki, Finland

$11{ }^{3}$ Gerontology Research Center, Faculty of Sport and Health Sciences, University of Jyväskylä,

12 Jyväskylä, Finland

$13{ }^{4}$ Department of Social Research, University of Helsinki, Helsinki, Finland

$14{ }^{5}$ Age Institute, Helsinki, Finland

$15{ }^{6}$ Hospital for Children and Adolescents, Helsinki University Hospital and University of Helsinki,

16 Helsinki, Finland

$17{ }^{7}$ PEDEGO Research Unit, MRC Oulu, Oulu University Hospital and University of Oulu, Oulu,

18 Finland

$19{ }^{8}$ Department of General Practice and Primary Health Care, University of Helsinki and Helsinki

20 University Hospital, Unit of General Practice, Helsinki, Finland

$21{ }^{9}$ Vaasa Central Hospital, Vaasa, Finland

Corresponding author: Mia-Maria Perälä, Department of Public Health Solutions, National Institute for Health and Welfare, P.O. Box 30, FI-00271 Helsinki, Finland, e-mail mia.perala@thl.fi

Key words: disability, Nordic diet, Mediterranean diet, mobility limitation, ageing

29 Running title: Healthy diet and disability

30 Word, reference, tables: the abstract 257, main text 2992, references 41 , tables 3 , appendix 31 table 1. 
34 This work was supported by grants from Academy of Finland, Finska Läkaresällskapet, Samfundet 35 Folkhälsan, Juho Vainio Foundation, Signe and Ane Gyllenberg Foundation, Yrjö Jahnsson

36 Foundation, Diabetes Research Foundation, Finnish Foundation for Cardiovascular Research. EU

37 H2020-PHC-2014-DynaHealth grant no. 633595. The Academy of Finland supported MBvB (grant 38 no. 257239); EK (grants no. 127437, 129306, 130326, 134791 and 263924), and JGE (grants no. $39129369,129907,135072,129255$ and 126775).

40

41 Conflict of interest

42 None. 
The healthy Nordic diet and Mediterranean diet and incidence of disability 10 years later in home-dwelling old adults

\section{Abstract}

Background/Objective: Diet has a major impact on a person's health. However, limited information exists on the long-term role of the whole diet on disability. We investigated the association of the healthy Nordic diet and the Mediterranean diet with incident disability 10 years 51 later.

52 Design: Longitudinal, with a follow-up of 10 years.

53 Settings/Participants: A total of 962 home-dwelling men and women from the Helsinki Birth

54 Cohort Study, mean age $61.6 \mathrm{y}$, who were free of disability at baseline.

55 Measurements: At baseline, 2001-2004, the Nordic diet score (NDS) and modified Mediterranean diet score (mMDS) were calculated using a validated 128-item food-frequency questionnaire.

57 Higher scores indicated better adherence to the diet. Participants' incident disability was assessed

58 during 2011-2013 by a self-reported questionnaire and was based on mobility limitations and 59 difficulties to perform self-care activities. Analyses were performed using logistic regression and 60 adjusted for potential confounding factors.

61 Results: In total, 94 participants (9.8\%) developed mobility limitations and 45 participants (4.7\%)

62 developed difficulties in self-care activities during 10 year follow-up. The likelihood of having 63 mobility limitations (odds ratio (OR) $0.42,95 \%$ confidence interval (CI) $0.22-0.80$ ) and difficulties

64 in self-care activities (OR 0.38, 95\% CI 0.15-0.94) were lower among those in the highest NDS 65 tertile than among those in the lowest NDS tertile. Greater mMDS was associated with a lower 66 disability incidence; however, the association was not statistically significant.

67 Conclusions/Implications: Adherence to the healthy Nordic diet predicts 10-year incidence of mobility limitations and difficulties to perform self-care activities in old age and may thus be protective against disability in Nordic population. 
02

\section{Introduction}

World Health Organization identifies disability in old individuals as people's difficulty to participate in daily activities or activities that are necessary for independent living and integration in their environment. ${ }^{(1)}$ There are several domains of disability, such as mobility limitations and difficulties in activities of daily living (ADL) or instrumental activities of daily living (IADL) that are essential for independent life. Studies have shown that mobility limitation is a strong predictor of ADL and IADL disability. ${ }^{(2)}$ It has been estimated that 8-35\% of individuals 65 years old or older have mobility limitations or difficulties in performing ADL tasks. ${ }^{(2-4)}$ Mobility limitations and difficulties to perform self-care activities increase the risk for hospitalization, nursing home admission and are strong predictors of mortality. ${ }^{(5,6)}$ Therefore, it is important to understand the processes that cause the progression of disability in order to develop strategies to prevent or delay disability.

Poor health and physical performance, smoking as well as cognitive impairment are well known risk factors for disability. ${ }^{(2,7)}$ Diet is also a potential modifiable factor affecting disability.

Observational studies have shown associations between low circulating levels of carotenoids and vitamin D and B12 ${ }^{(8,9)}$ as well as low intakes of single antioxidant nutrients, fruits and vegetables $(10,11)$ and mobility limitations and ADL. However, despite the previously described studies concerning single dietary factors and disability, the role of the whole diet in disablement process is still mostly unknown. A few studies have shown that adherence to Healthy Eating Index ${ }^{(12,13)}$ and Japanese diet ${ }^{(14)}$ are related to a lower likelihood of mobility limitations. In addition, Mediterranean diet has been shown to decrease the risk of disability and slow the decline of mobility and walking speed. ${ }^{(15-18)}$ We have recently observed a positive relationship between the healthy Nordic diet and overall physical performance and muscle strength. ${ }^{(19,20)}$ However, no studies exist in which the association of the healthy Nordic diet with incidence of disability has been examined. In addition, previous studies have not been investigated and compared different diet scores in the same cohort with respect to incidence of disability. Investigating different diet scores may help in identifying which diet is appropriate for population concerned. Therefore, we investigated whether two diet scores, the Nordic diet and the modified Mediterranean diet scores, predicts mobility limitations and difficulties to perform self-care activities 10 years later in initially healthy home-dwelling men and women aged 61 years on average at baseline.

\section{Methods}

Design and Study Population 
104 The subjects in this study belong to the Helsinki Birth Cohort Study which originally included 4630 105 men and 4130 women who were born as singletons at Helsinki University Central Hospital between 1061934 and 1944. ${ }^{(21)}$ A random sample of 2902 individuals were invited to participate in a clinical 107 examination conducted between 2001 and 2004. Of these, 2003 men $(n=928)$ and women $(n=$ 108 1075) participated in the examination. From 2011-2013, 1404 subjects were still traceable. Of 109 these, 1094 participants (478 men and 616 women) attended a clinical examination between 2011 110 and 2013. ${ }^{(19)}$ Those who participated in the follow-up clinical examination had lower rates of 111 mobility limitations (6.2\% v. $16.6 \%)$ and difficulties in self-care activities (4.6\% v. $12.1 \%)$ at 112 baseline compared to those who did not participate. Participants who had adequate data on diet and 113 disability, and were free of disability at baseline were included in this study $(n=962)$.

115 This study was conducted according to the guidelines laid down in the Declaration of Helsinki and

116 all procedures involving human subjects were approved by the Ethics Committee of the Helsinki 117 and Uusimaa Hospital District. Written informed consent was obtained from all subjects.

\section{Dietary Assessment}

120 At the baseline clinical examination, diet was assessed by a validated, self-administered, 128-item 121 food-frequency questionnaire (FFQ). ${ }^{(22,23)}$ Participants were asked how often, on average, they consumed each food item in the past year. The frequency responses ranged from "never or seldom" to "at least six times a day". The portion size was fixed for each food item or mixed dish (e.g. slice and glass) based on the national Findiet Study and present the most commonly used portion sizes in Finland. At the clinic, a research nurse checked the FFQ. The final decision regarding the completeness of the FFQ was made by a nutritionist. Exclusions were made due to incompletely filled FFQ and daily energy intake cut-off points corresponding to $0.5 \%$ at both ends of the daily energy intake distributions for men and women. ${ }^{(24)}$ The average daily intakes of nutrients and foods were calculated with the national food composition database Fineli®.

131 Details of the Nordic diet score (NDS), which is also known as the Baltic Sea diet score, and the 132 modified Mediterranean diet score (mMDS) are described in detail elsewhere. ${ }^{(25,26)}$ Briefly, the 133 NDS is based on 9 components. Favourable food components (Nordic vegetables (excluding 134 potatoes), Nordic fruits, Nordic cereals, fish, a ratio of polyunsaturated fatty acids to saturated and trans-fatty acids, and low-fat milk) were assigned values of 0-3 according to ascending sex-specific

136 quartile ranks, and unfavourable food components (red and processed meat, and intake of total fat) were assigned values of 0-3 according to descending sex-specific quartile ranks. In addition, men 
138 consuming $\leq 20 \mathrm{~g} /$ day and women consuming $\leq 10 \mathrm{~g} /$ day of alcohol received 1 point; otherwise, a 0 139 point was given. The mMDS is based on 9 components. For vegetables (excluding potatoes), fruit 140 and nuts, cereals, legumes, fish and fish products, and the ratio of monounsaturated and 141 polyunsaturated to saturated fat, 1 point was given if intake was above the sex-specific median, 0 142 otherwise. For meat and dairy products, 1 point was given if intake was below the sex-specific 143 median, 0 otherwise. For alcohol, 1 point was given if intake was between 10-50 g/day in men and $1445-25 \mathrm{~g} /$ day in women; otherwise, a 0 point was given. The NDS ranged from 0-25 and the mMDS 145 from 0-9.

Disability

148 Disability was assessed with the Finnish validated version of the RAND 36-Item Health Survey 1.0 149 (Short Form 36 [SF-36]) ${ }^{(27)}$ at baseline and follow-up clinical examination. The question on 150 difficulties in walking $500 \mathrm{~m}$ was used to assess mobility limitations and difficulties to perform 151 self-care activities was assessed with questions on difficulties in dressing or bathing. Response 152 categories were: no difficulties; little difficulties; many difficulties. Participants were considered to 153 have mobility limitations if they reported any difficulties in walking $500 \mathrm{~m}$ and difficulties to 154 perform self-care activities if they reported any difficulties in dressing and/or bathing.

156 For comparing whether self-reported mobility limitations is valid and identify those with worse 157 physical performance, participants' physical performance was tested at follow-up clinical 158 examination with the validated Senior Fitness Test ${ }^{(28)}$ and total score was calculated as described 159 earlier. ${ }^{(19)}$

\section{Covariates}

162 During the baseline clinical examination, height was measured to the nearest $0.1 \mathrm{~cm}$ and weight to $1630.1 \mathrm{~kg}$. BMI was calculated as weight (in $\mathrm{kg}$ ) divided by height (in $\mathrm{m}^{2}$ ). At the baseline clinical 164 examinations, the level of educational attainment (elementary school; vocational school; senior high 165 school and college; university degree), smoking status (never; former; current), presence of main 166 chronic diseases (cancer; cerebrovascular disease; hypertension; diabetes) were asked using questionnaire. Energy intake was measured with an FFQ as an average intake (in MJ per day).

168 Participants' physical activity over the previous 12 months was assessed using a validated exercise questionnaire. ${ }^{(29)}$ Depressive symptoms were assessed using the Beck depression inventory (BDI) (30), and participants with score $\geq 10$ were classified as having at least mild depression. Cognitive 
171 status was assessed by Mini-Mental State Examination (MMSE) ${ }^{(31)}$ score (0-30) during 2009-2011, 172 and participants with a score $<24$ were classified as having impaired cognition.

Statistical Methods

175 There were no significant interactions for the healthy diet and sex on disability, therefore, men and

176 women were analysed together. Descriptive characteristics were reported as mean (standard

177 deviation), proportions, or median (interquartile ranges) values, and the differences of baseline 178 characteristics across tertiles were tested with one-way ANOVA or Chi-square test. The association 179 between tertiles of NDS and mMDS and likelihood of having mobility limitations or difficulties to 180 perform self-care activities were examined by logistic regression models. The relationships between 181 each of the NDS and mMDS components and self-reported disability were also examined using 182 logistic regression models. Model 1 was adjusted for age and sex. To investigate whether diet was 183 independently related to disability, the Model 2 was further adjusted for potential confounding 184 factors related to disability or diet: BMI, physical activity, smoking status, educational attainment, 185 energy intake, and presence of chronic diseases and depression. ${ }^{(2,32,33)}$ Model 3 was further 186 adjusted for cognitive status and included only those participants whose MMSE test result was 187 available ( $\mathrm{n}=832$ ). All statistical analyses were done using the SPSS Statistics version 23 for 188 Windows ${ }^{\circledR}$ (SPSS Inc., Chicago, IL, USA). Significance was defined as $\mathrm{P}<.05$.

\section{Results}

191 The basic characteristics of the study population for men $(n=439)$ and women $(n=523)$ are seen in 192 Table 1 and daily intakes of NDS and mMDS components in Appendix Table 1. The correlation between NDS and mMDS was $0.56(\mathrm{P}<.001)$. Incident mobility limitation was more common than self-care dependence. During a 10-year follow-up, 94 participants (9.8\%) developed mobility limitations and 45 participants (4.7\%) developed difficulties to perform self-care activities. Senior Fitness Test total score was significantly smaller among those with mobility limitations (32.9 points, SD 15.1) than those with no limitations (47.9 points, SD 16.6).

After adjusting for age, BMI, educational attainment, physical activity, smoking status, energy intake, and the presence of chronic disease and depression the likelihood of having difficulties to walk $500 \mathrm{~m}$ were lower among those in the highest NDS tertile than among those in the lowest NDS tertile (odds ratio (OR) $0.42,95 \%$ confidence interval (CI) $0.22-0.80$ ) (Table 2). In addition, individuals with the highest adherence to a healthy Nordic diet at baseline had lower likelihood of developing difficulties to perform self-care activities compared with those with the lowest 
adherence (OR 0.38, 95\% CI 0.15-0.94). In total, 832 participants had completed MMSE test results. Including cognitive status to the model, the likelihood of having disability were lower among those with high NDS, however, only the association between the NDS and mobility limitations remained significant.

After adjusting for age and sex the likelihood of having mobility limitation (OR 0.65, 95\% CI 0.351.22 ) and difficulties to perform self-care activities (OR $0.59,95 \%$ CI $0.26-1.35)$ was lower among those who had the highest mMDS compared to those who had the lowest mMDS, however, the associations did not reach statistical significance (Table 2). Adjusting for further potential confounders did not have a major effect on the results.

When investigating the associations between NDS and mMDS components and disability, we found statistical significant associations of greater intake of fat and smaller intake of cereals with increased likelihood of having difficulties to perform self-care activities (Table 3). Greater intake of alcohol was significantly related to lower likelihood of having mobility limitations.

\section{Discussion}

In our study of 962 home-dwelling ageing men and women, we observed that the healthy Nordic diet is a strong predictor of two states of disability; mobility limitations and difficulties to perform self-care activities, even after adjusting for potential confounding factors including physical activity, chronic diseases and depression. Adherence to the Mediterranean diet was also related to lower likelihood of being disabled, however, the association was not statistically significant. To our knowledge, there are no previous studies that have compared different healthy diets within the same cohort for disability outcomes. Our findings extend the relevance of a healthy diet to target mobility limitations and difficulties to perform self-care activities and support the importance of diet in the pathogenesis of disability in old age.

Our results support the previous findings in which the protective effect of healthy diet, including the Healthy Eating Index, Japanese diet and Mediterranean diet, on disability has been observed. ${ }^{(12-16)}$ Even though these diets vary in food and nutrient components, they all capture essential elements of a high-quality diet including high consumption of fruits, vegetables, and fish and low consumption of red and processed meat. When we investigated single food components of NDS and mMDS, we observed that only intake of fat, alcohol and cereals were related to disability. Based on our results, we suggest that the whole diet is a stronger predictor of disability in old age than single foods or 
nutrients. Therefore, the findings in our study extend the previous results and suggest that healthy diet, which include high amounts of fruit, vegetables, and fish, moderate intake of alcohol, and small amounts of red meat and fat, help to prevent disability in old age.

Even though the healthy Nordic and Mediterranean diet were related to lower likelihood of disability, only the association of healthy Nordic diet with disability was significant. The healthy Nordic diet contains locally grown healthy Nordic foods whereas the widely investigated Mediterranean diet contains foods familiar in the Mediterranean area such as seeds, nuts and olive oil. Therefore, adherence to the healthy Nordic diet may be easier in Nordic population. The major difference between these diets is that dairy products are beneficial in the NDS, whereas they are detrimental in the mMDS. In addition, as these diet scores are based on study specific cut-offs for each food components, the absolute intake of each component may differ between studies even though diet scores are identical. Indeed, we observed that the median consumption of food components included in the mMDS, such as fruits and vegetables, were in part lower than observed in previous studies ${ }^{(15,18)}$, suggesting that the diet of our study population is not as healthy than in the Mediterranean area. Furthermore, it should be noted that there are several different versions of Mediterranean diet score, which make it difficult to compare the results. Moreover, the range of the MDS is much smaller (0-9) compared to the NDS (0-25) which may limit the statistical power. Therefore, we propose that these could partly explain why we did not observe as strong associations between Mediterranean diet and disability as with healthy Nordic diet.

Strong evidence exist that mobility limitations are the results of physical impairments, such as decreased muscle strength ${ }^{(34,35)}$. Several diseases and changes in body homeostasis also affect muscle strength and thus mobility. Previously, it has been demonstrated that adherence to the healthy Nordic and Mediterranean diet predicts greater muscle strength and thus better overall physical performance. ${ }^{(16,18-20)}$ In addition, both of these diets have been found to be associated with a lower risk of several diseases, such as diabetes and cardiovascular disease, and changes in body homeostasis including insulin resistance and inflammation. ${ }^{(36-39)}$ Therefore, we suggest that plausible explanations for the observed associations between the healthy diet and disability are multifactorial, including beneficial effects of diet on muscle strength and on risk factors of chronic diseases. In addition, adherence to the healthy diet may also be linked with overall healthy lifestyles practices including increased physical activity, which associates with disability. 
272 A significant strength of our study is its longitudinal study design that provides opportunity to 273 investigate long-term influences of diet on disability. In addition, our study included a large study 274 population consisting of both men and women. A further strength of our study is that we measured 275 two states of disability; difficulties to perform self-care activities, which is more serious and 276 mobility limitations, which are more common. Our study also has some limitations as discussed 277 earlier. ${ }^{(20)}$ Firstly, we adjusted for a multitude of covariates, including physical activity, 278 socioeconomic status and chronic diseases; however, potential unmeasured and residual 279 confounding cannot be excluded. Secondly, diet was measured by a validated FFQ ${ }^{(22,23)}$ only at 280 baseline; therefore changes in the diet were not captured. Thirdly, the disability status of 281 participants was self-reported, which may cause misclassification of disability status. ${ }^{(3)}$ However, it 282 has been shown that self-reported difficulties in walking reflect people's physical performance 283 status and is able to identify those with worse physical performance. ${ }^{(40,41)}$ Therefore, it is valid and 284 has clinical significance. We also observed that those who reported mobility limitations had 285 significantly poorer physical performance than those with no limitations. In addition, we measured 286 only one of the ADL components, difficulty to bath and dress, which may underestimate the 287 difficulties in self-care activities. However, difficulty to bath is the most common ADL disability and it represents the first ADL in which most persons become disabled. Therefore, we suggest that it is a good indicator of ADL. Further, as reported earlier, those individuals who participated in the follow-up clinical examination had lower rates of disability, were younger, thinner, more educated, and had healthier diet at baseline compared with those who did not participate. ${ }^{(19)}$ Those with unhealthy lifestyle habits may have died at earlier ages or developed disability and were, therefore, excluded from the study. Selective survival may thus have influenced the association between the healthy diet and disability. In addition, the incidence of disability was low during a 10-year followup which may limit the statistical power. Thus, participants may not be fully representative of all older people living in Finland.

299 Our study indicates that adherence to the healthy Nordic diet decreased the likelihood of mobility 300 limitations and difficulties to perform self-care activities in old age. In addition, Mediterranean diet may also have a beneficial impact on disability. Our results may have important clinical implications. As disability is common among older age and restricts a person's independence as well as affects overall health, it is important try to prevent dis ability. Our results indicates that adherence to healthy diet provide one opportunity to prevent or delay disability. In addition, it is well known that adherence to healthy diet has several other health benefits as well. Therefore, 
306 adhering to a healthy diet may have major beneficial effect on people's health. However, future

307 intervention studies as well as longitudinal studies, that compare different diet scores with incidence 308 of disability, are critically needed.

309 


\section{References}

311 1. WHO (World Health Organization). International Classification of Functioning,

312 Disability and Health (ICF). Geneva: WHO (World Health Organization); 2001.

313 2. Guralnik JM, Fried LP, Salive ME. Disability as a public health outcome in the aging

314 population. Annu Rev Public Health 1996;17:25-46.

315 3. Sainio P, Koskinen S, Heliövaara M, et al. Self-reported and test-based mobility

316 limitations in a representative sample of Finns aged 30+. Scand J Public Health 2006;34:378-

317386.

318 4. Shumway-Cook A, Ciol MA, Yorkston KM, et al. Mobility limitations in the Medicare

319 population: prevalence and sociodemographic and clinical correlates. J Am Geriatr Soc

$320 \quad$ 2005;53:1217-1221.

321 5. Leskinen R, Laatikainen T, Peltonen M, et al. Self-reported walking difficulty predicts

322 late-life mortality in Finnish war veterans: results from the Veteran 1992 Project Survey. J

323 Am Geriatr Soc 2015;63:118-123.

324 6. Wu LW, Chen WL, Peng TC, et al. All-cause mortality risk in elderly individuals with

325 disabilities: a retrospective observational study. BMJ Open 2016;6:e011164.

326 7. Legrand D, Vaes B, Matheï C, et al. Muscle strength and physical performance as

327 predictors of mortality, hospitalization, and disability in the oldest old. J Am Geriatr Soc

328 2014;62:1030-1038.

329 8. Bartali B, Semba RD, Frongillo EA, et al. Low micronutrient levels as a predictor of

330 incident disability in older women. Arch Intern Med 2006;166:2335-2340.

331 9. Houston DK, Neiberg RH, Tooze JA, et al. Low 25-hydroxyvitamin D predicts the onset

332 of mobility limitation and disability in community-dwelling older adults: the Health ABC

333 Study. J Gerontol A Biol Sci Med Sci 2013;68:181-187.

334 10. Houston DK, Stevens J, Cai J, et al. Dairy, fruit, and vegetable intakes and functional

335 limitations and disability in a biracial cohort: the Atherosclerosis Risk in Communities

336 Study. Am J Clin Nutr 2005;81:515-522.

337 11. Tomey KM, Sowers MR, Crandall C, et al. Dietary intake related to prevalent functional

338 limitations in midlife women. Am J Epidemiol 2008;167:935-943.

339 12. Koster A, Penninx BW, Newman AB, et al. Lifestyle factors and incident mobility

340 limitation in obese and non-obese older adults. Obesity (Silver Spring) 2007;15:3122-3132.

341 13. Xu B, Houston D, Locher JL, et al. The association between Healthy Eating Index-2005

342 scores and disability among older Americans. Age Ageing 2012;41:365-371. 
343 14. Tomata Y, Watanabe T, Sugawara Y, et al. Dietary patterns and incident functional

344 disability in elderly Japanese: the Ohsaki Cohort 2006 study. J Gerontol A Biol Sci Med Sci

345 2014;69:843-851.

346 15. Féart C, Pérès K, Samieri C, et al. Adherence to a Mediterranean diet and onset of

347 disability in older persons. Eur J Epidemiol 2011;26:747-756.

348 16. Milaneschi Y, Bandinelli S, Corsi AM, et al. Mediterranean diet and mobility decline in 349 older persons. Exp Gerontol 2011;46:303-308.

350 17. Shahar DR, Houston DK, Hue TF, et al. Adherence to mediterranean diet and decline in 351 walking speed over 8 years in community-dwelling older adults. J Am Geriatr Soc 352 2012;60:1881-1888.

353 18. Zbeida M, Goldsmith R, Shimony T, et al. Mediterranean diet and functional indicators 354 among older adults in non-Mediterranean and Mediterranean countries. J Nutr Health Aging $355 \quad 2014 ; 18: 411-418$.

356 19. Perälä MM, von Bonsdorff M, Männistö S, et al. A healthy Nordic diet and physical 357 performance in old age: findings from the longitudinal Helsinki Birth Cohort Study. Br J 358 Nutr 2016;115:878-886.

359 20. Perälä MM, von Bonsdorff MB, Männistö S, et al. The healthy Nordic diet predicts 360 muscle strength 10 years later in old women, but not old men. Age Ageing 2017;46:588-594.

361 21. Barker DJ, Osmond C, Forsén TJ, et al. Trajectories of growth among children who have 362 coronary events as adults. N Engl J Med 2005;353:1802-1809.

363 22. Männistö S, Virtanen M, Mikkonen T, et al. Reproducibility and validity of a food 364 frequency questionnaire in a case-control study on breast cancer. J Clin Epidemiol 365 1996;49:401-409.

366 23. Paalanen L, Männistö S, Virtanen MJ, et al. Validity of a food frequency questionnaire 367 varied by age and body mass index. J Clin Epidemiol 2006;59:994-1001.

368 24. Meltzer HM, Brantsaeter AL, Ydersbond TA, et al. Methodological challenges when 369 monitoring the diet of pregnant women in a large study: experiences from the Norwegian 370 Mother and Child Cohort Study (MoBa). Mat Child Nutr 2008;4:14-27.

371 25. Kanerva N, Kaartinen NE, Schwab U, et al. The Baltic Sea Diet Score: a tool for 372 assessing healthy eating in Nordic countries. Public Health Nutr 2013;17:1697-1705.

373 26. Trichopoulou A, Orfanos P, Norat T, et al. Modified Mediterranean diet and survival:

374 EPIC-elderly prospective cohort study. BMJ 2005;330:991.

375 27. Ware JE Jr, Sherbourne CD. The MOS 36-item short-form health survey (SF-36). I.

376 Conceptual framework and item selection. Med Care 1992;30:473-483. 
377 28. Rikli RE, Jones CJ. Development and validation of a functional fitness test for 378 community-residing older adults. J Aging Phys Act 1999;6:127-159.

379 29. Lakka TA, Salonen JT. Intra-person variability of various physical activity assessments

380 in the Kuopio Ischaemic Heart Disease Risk Factor Study. International journal of 381 epidemiology 1992;21:467-472.

382 30. Beck AT, Steer RA, Garbin MG. Psychometric properties of the Beck Depression 383 Inventory: Twenty-five years of evaluation. Clin Psychol Rev 1988;8:77-100.

384 31. Folstein MF, Folstein SE, McHugh PR. "Mini-mental state". A practical method for 385 grading the cognitive state of patients for the clinician. J Psychiatr Res 1975;12:189-198.

386 32. Maynard M, Gunnell D, Ness AR, et al. What influences diet in early old age?

387 Prospective and cross-sectional analyses of the Boyd Orr cohort. Eur J Public Health 388 2006;16: 316-24.

389 33. Thiele S, Mensink GB, Beitz R. Determinants of diet quality. Public Health Nutr 2004;7: $390 \quad 29-37$.

391 34. Stenholm S, Shardell M, Bandinelli S, et al. Physiological factors contributing to 392 mobility loss over 9 years of follow-up-results from the InCHIANTI study. J Gerontol A Biol 393 Sci Med Sci 2015;70:591-597.

394 35. Visser M, Goodpaster BH, Kritchevsky SB, et al. Muscle mass, muscle strength, and 395 muscle fat infiltration as predictors of incident mobility limitations in well-functioning older 396 persons. J Gerontol A Biol Sci Med Sci 2005;60:324-333.

397 36. Kanerva N, Kaartinen NE, Rissanen H, et al. Associations of the Baltic Sea diet with 398 cardiometabolic risk factors--a meta-analysis of three Finnish studies. Br J Nutr 399 2014;112:616-626.

400 37. Kastorini CM, Milionis HJ, Esposito K, et al. The effect of Mediterranean diet on 401 metabolic syndrome and its components: a meta-analysis of 50 studies and 534,906

402 individuals. Journal of the American College of Cardiology 2011;57:1299-1313.

403 38. Lacoppidan SA, Kyrø C, Loft S, et al. Adherence to a Healthy Nordic Food Index Is 404 Associated with a Lower Risk of Type-2 Diabetes -The Danish Diet, Cancer and Health 405 Cohort Study. Nutrients 2015;7:8633-8644.

406 39. Sofi F, Macchi C, Abbate R, et al. Mediterranean diet and health status: an updated 407 meta-analysis and a proposal for a literature-based adherence score. Public Health Nutr $408 \quad 2014 ; 17: 2769-2782$. 
409 40. Fried LP, Young Y, Rubin G, et al. Self-reported preclinical disability identifies older 410 women with early declines in performance and early disease. J Clin Epidemiol 2001;54:889411901.

412 41. Wang CY, Hu MH, Chen HY, Li RH. Self-reported mobility and instrumental activities 413 of daily living: Test-retest reliability and criterion validity. J Aging Phys Act 2012;20:186414197.

415 
416 Table 1. Characteristics of the subjects by adherence to the diet score tertile

\begin{tabular}{|c|c|c|c|c|c|c|c|c|c|c|c|c|c|c|}
\hline \multirow{3}{*}{ n (men \%) } & \multicolumn{7}{|c|}{ Nordic diet score } & \multicolumn{7}{|c|}{ modified Mediterranean diet score } \\
\hline & \multicolumn{2}{|c|}{ Low $(0-11)$} & \multicolumn{2}{|c|}{ Middle (12-15) } & \multicolumn{2}{|c|}{ High (16-25) } & \multirow{2}{*}{$\frac{\mathrm{P}}{.28}$} & \multicolumn{2}{|c|}{ Low $(0-3)$} & \multicolumn{2}{|c|}{ Middle (4-5) } & \multicolumn{2}{|c|}{ High (6-9) } & \multirow{2}{*}{$\begin{array}{c}\mathrm{P} \\
.83\end{array}$} \\
\hline & 366 & $(48.4)$ & 308 & $(42.2)$ & 288 & $(45.8)$ & & 284 & $(45.8)$ & 423 & $(44.7)$ & 255 & $(47.1)$ & \\
\hline Age (years) $*$ & 61.0 & 2.7 & 61.3 & 2.8 & 61.4 & 2.7 & .25 & 61.3 & 2.9 & 61.2 & 2.8 & 61.2 & 2.5 & .81 \\
\hline Body mass index $\left(\mathrm{kg} / \mathrm{m}^{2}\right) *$ & 26.9 & 3.9 & 26.9 & 3.8 & 26.9 & 4.0 & .97 & 27.0 & 3.8 & 26.9 & 3.9 & 26.7 & 3.9 & .60 \\
\hline Physical activity (MET h/week) * & 34.9 & 23.4 & 38.7 & 29.0 & 42.1 & 24.3 & $<.001$ & 36.5 & 26.4 & 36.8 & 23.8 & 42.9 & 27.6 & .004 \\
\hline Energy intake $(\mathrm{MJ}) *$ & 8.5 & 2.9 & 9.4 & 3.3 & 10.0 & 3.1 & $<.001$ & 8.1 & 2.7 & 9.2 & 3.1 & 10.5 & 3.4 & $<.001$ \\
\hline Chronic diseases $(\%) *, \dagger$ & \multicolumn{2}{|c|}{37.5} & \multicolumn{2}{|c|}{41.0} & \multicolumn{2}{|c|}{41.3} & .50 & \multicolumn{2}{|c|}{38.4} & \multicolumn{2}{|c|}{40.1} & \multicolumn{2}{|c|}{40.8} & .34 \\
\hline Self-care dependence $(\%) \|, \mathscr{I}$ & & 5.7 & & & & .4 & .097 & & 6.0 & & .5 & & 3.5 & .39 \\
\hline
\end{tabular}

417 MET, metabolic equivalent.

418 Data are expressed as mean values (with standard deviations) for continuous values or percentages for categorical values. The differences

419 between the diet score groups were tested with one-way ANOVA for continuous variables and Chi-square test for categorical variables.

$420 *$ At baseline clinical examination.

$421 \uparrow$ Smoking one or more cigarettes per day. 
422 \$ Presence of at least 1 chronic disease.

$423 \S$ Having at least mild depression.

424 || At follow-up clinical examination.

425 II Difficulties to perform self-care activities. 
427 Table 2. Odds ratios of mobility limitations and difficulties to perform self-care activities

428 over 10-year follow-up for baseline adherence to Nordic and Mediterranean diet among

429 Helsinki Birth Cohort Study participants (n=962)

\begin{tabular}{|c|c|c|c|c|c|c|c|c|c|}
\hline \multirow{3}{*}{\multicolumn{2}{|c|}{$\begin{array}{l}\text { OR } \\
\text { Mobility limitations }\end{array}$}} & \multicolumn{2}{|l|}{ Model $1 *$} & \multicolumn{3}{|c|}{ Model $2 \dagger$} & \multicolumn{3}{|c|}{ Model 3 t } \\
\hline & & $95 \% \mathrm{CI}$ & $\mathrm{P} \S$ & OR & $95 \% \mathrm{CI}$ & $\mathrm{P} \S$ & OR & $95 \% \mathrm{CI}$ & $\mathrm{P} \S$ \\
\hline & & & & & & & & & \\
\hline \multicolumn{10}{|c|}{ NDS tertiles } \\
\hline Low & Ref. & & & Ref. & & & Ref. & & \\
\hline Middle & 0.86 & $0.54-1.39$ & & 0.88 & $0.53-1.48$ & & 0.81 & $0.46-1.40$ & \\
\hline High & 0.42 & $0.23-0.76$ & .005 & 0.42 & $0.22-0.80$ & .010 & 0.42 & $0.21-0.84$ & .014 \\
\hline \multicolumn{10}{|c|}{ mMDS tertiles } \\
\hline Low & Ref. & & & Ref. & & & Ref. & & \\
\hline Middle & 1.21 & $0.74-1.97$ & & 1.15 & $0.68-1.98$ & & 1.15 & $0.65-2.01$ & \\
\hline High & 0.65 & $0.35-1.22$ & .12 & 0.69 & $0.34-1.37$ & .26 & 0.61 & $0.28-1.30$ & .16 \\
\hline \multicolumn{10}{|c|}{ Difficulties to perform self-care activities } \\
\hline \multicolumn{10}{|c|}{ NDS tertiles } \\
\hline Low & Ref. & & & Ref. & & & Ref. & & \\
\hline Middle & 0.92 & $0.47-1.78$ & & 0.89 & $0.45-1.79$ & & 0.87 & $0.41-1.86$ & \\
\hline High & 0.39 & $0.16-0.93$ & .039 & 0.38 & $0.15-0.94$ & .039 & 0.45 & $0.17-1.16$ & .087 \\
\hline \multicolumn{10}{|c|}{ mMDS tertiles } \\
\hline Low & Ref. & & & Ref. & & & Ref. & & \\
\hline Middle & 0.75 & $0.38-1.47$ & & 0.60 & $0.30-1.23$ & & 0.60 & $0.28-1.27$ & \\
\hline High & 0.59 & $0.26-1.35$ & .21 & 0.51 & $0.21-1.25$ & .17 & 0.45 & $0.17-1.21$ & .12 \\
\hline
\end{tabular}

430 OR, odds ratio; CI, confidence interval; NDS, Nordic diet score; mMDS, modified

431 Mediterranean diet score; Ref., referent value.

$432 *$ Model 1, adjusted for sex and age.

$433 \uparrow$ Model 2, adjusted for Model 1 plus body mass index, educational attainment, smoking

434 status, physical activity, energy intake, presence of chronic diseases and depression.

$435 \ddagger$ Model 3, adjusted for Model 2 plus impaired cognitive function $(n=832)$.

$436 \S \mathrm{P}$ for a linear trend. 
438 Table 3. Association between sex specific tertiles of Nordic and Mediterranean diet score components and risk of mobility limitations and

439 difficulties to perform self-care activities during a 10 year follow-up of old adults $(n=962)$ *

\begin{tabular}{|c|c|c|c|c|c|c|c|c|c|c|}
\hline \multirow[b]{3}{*}{ Component (g/d) } & \multicolumn{5}{|c|}{ Mobility limitations } & \multicolumn{5}{|c|}{ Difficulties in self-care activities } \\
\hline & \multirow[t]{2}{*}{1} & \multicolumn{2}{|c|}{2} & \multicolumn{2}{|c|}{3} & \multirow[t]{2}{*}{1} & \multicolumn{2}{|c|}{2} & \multicolumn{2}{|c|}{3} \\
\hline & & OR & $95 \% \mathrm{CI}$ & OR & $95 \% \mathrm{CI}$ & & OR & $95 \% \mathrm{CI}$ & OR & $95 \% \mathrm{CI}$ \\
\hline \multicolumn{11}{|l|}{ NDS component } \\
\hline Nordic vegetables & Ref. & 0.95 & $0.53-1.70$ & 0.76 & $0.39-1.47$ & Ref. & 0.64 & $0.28-1.47$ & 0.99 & $0.42-2.35$ \\
\hline Nordic fruits & Ref. & 0.94 & $0.52-1.71$ & 0.94 & $0.49-1.78$ & Ref. & 1.26 & $0.56-2.82$ & 1.30 & $0.53-3.21$ \\
\hline Nordic cereals & Ref. & 0.62 & $0.34-1.12$ & 0.77 & $0.42-1.44$ & Ref. & 0.91 & $0.42-1.97$ & 0.64 & $0.27-1.50$ \\
\hline Fat intake (E\%) & Ref. & 2.12 & $1.11-4.05$ & 1.93 & $0.94-3.97$ & Ref. & 3.52 & $1.21-10.24$ & 4.13 & $1.33-12.84 \dagger$ \\
\hline Fat ratio $\$$ & Ref. & 1.03 & $0.57-1.88$ & 1.14 & $0.60-2.18$ & Ref. & 0.98 & $0.45-2.13$ & 0.65 & $0.26-1.62$ \\
\hline Low-fat milk & Ref. & 1.80 & $1.02-3.20$ & 1.03 & $0.54-1.94$ & Ref. & 2.36 & $1.02-5.45$ & 1.59 & $0.63-4.00$ \\
\hline Fish & Ref. & 1.00 & $0.57-1.76$ & 0.74 & $0.38-1.47$ & Ref. & 0.50 & $0.22-1.16$ & 0.68 & $0.28-1.65$ \\
\hline Red meat & Ref. & 1.16 & $0.62-2.16$ & 1.09 & $0.54-2.19$ & Ref. & 2.58 & $1.01-6.60$ & 1.28 & $0.44-3.72$ \\
\hline Alcohol $\S$ & Ref. & 0.77 & $0.45-1.35$ & 0.48 & $0.25-0.91 \dagger$ & Ref. & 1.21 & $0.54-2.70$ & 1.02 & $0.42-2.45$ \\
\hline \multicolumn{11}{|l|}{ mMDS component } \\
\hline All vegetables & Ref. & 0.53 & $0.29-0.98$ & 0.80 & $0.42-1.51$ & Ref. & 0.46 & $0.18-1.17$ & 1.82 & $0.79-4.19$ \\
\hline All fruits and nuts & Ref. & 0.71 & $0.39-1.30$ & 0.55 & $0.29-1.05$ & Ref. & 1.38 & $0.61-3.15$ & 0.88 & $0.36-2.15$ \\
\hline All cereals & Ref. & 0.66 & $0.35-1.25$ & 0.74 & $0.36-1.49$ & Ref. & 0.54 & $0.24-1.22$ & 0.26 & $0.09-0.73 \dagger$ \\
\hline
\end{tabular}




\begin{tabular}{lllllllllll}
\hline Legumes & Ref. & 0.58 & $0.32-1.07$ & 0.94 & $0.52-1.70$ & Ref. & 1.21 & $0.56-2.60$ & 0.59 & $0.24-1.43$ \\
All dairy products & Ref. & 0.78 & $0.43-1.42$ & 0.81 & $0.44-1.50$ & Ref. & 1.11 & $0.48-2.53$ & 1.07 & $0.46-2.49$ \\
Fat ratio \| & Ref. & 0.74 & $0.40-1.36$ & 0.99 & $0.53-1.87$ & Ref. & 0.63 & $0.28-1.41$ & 0.49 & $0.20-1.21$ \\
Fish II & Ref. & 1.76 & $0.99-3.10$ & 0.94 & $0.48-1.86$ & Ref. & 1.10 & $0.50-2.41$ & 0.91 & $0.36-2.25$ \\
Meat ** & Ref. & 1.31 & $0.71-2.43$ & 1.13 & $0.56-2.27$ & Ref. & 3.11 & $1.24-7.78$ & 2.29 & $0.80-6.61$ \\
Alcohol § & Ref. & 0.74 & $0.43-1.28$ & 0.45 & $0.24-0.85 \dagger$ & Ref. & 1.14 & $0.52-2.50$ & 0.78 & $0.33-1.85$
\end{tabular}

440 OR, odds ratio; CI, confidence interval; NDS, Nordic diet score; mMDS, modified Mediterranean diet score; Ref., referent value.

$441 *$ Adjusted for sex, age, body mass index, educational attainment, smoking status, physical activity, energy intake, presence of chronic diseases

442 and depression, and mutually adjusted for the diet score components.

$443 \dagger \mathrm{P}$ for a linear trend $<.05$.

$444+$ A ratio of polyunsaturated fatty acids to saturated and trans-fatty acids.

$445 \S$ Both diet scores included alcohol, calculated as $100 \%$ ethanol.

$446 \|$ A ratio of unsaturated fatty acids to saturated fatty acids.

447 II All fish and fish products.

$448 \quad * *$ All meat and meat products. 
450 Appendix Table 1. Daily intakes of healthy Nordic diet score and modified Mediterranean

451 diet score components by sex in 962 Helsinki Birth Cohort Study participants

452

\begin{tabular}{|c|c|c|}
\hline Component (g/day) & Men & Women 453 \\
\hline NDS component & & 454 \\
\hline Nordic vegetables & $179(121,268)$ & $231(160,334) 55$ \\
\hline Nordic fruits & $84(30,161)$ & $116(58,207456$ \\
\hline Nordic cereals & $58(31,81)$ & $54(30,75)^{457}$ \\
\hline Fat intake $(\mathrm{E} \%)$ & $33(29,36)$ & $32(29,36) \quad 458$ \\
\hline Fat ratio $*$ & $0.4(0.4,0.5)$ & $0.4(0.3,0.5)^{459}$ \\
\hline Low-fat milk & $153(13,412)$ & $86(11,221)^{460}$ \\
\hline Fish & $45(29,64)$ & $33(21,45)_{462}^{401}$ \\
\hline Red and processed meat & $128(87,178)$ & $80(54,119)_{463}$ \\
\hline Alcohol $\dagger$ & $11(4,19)$ & $3(2,7) \quad 464$ \\
\hline mMDS component & & 465 \\
\hline All vegetables & $217(149,324)$ & $275(191,3924) 66$ \\
\hline All fruits and nuts & $206(93,331)$ & $263(147,4284) 67$ \\
\hline All cereals & $151(108,198)$ & $131(97,164) 468$ \\
\hline Legumes & $9(4,14)$ & $8(4,12) 469$ \\
\hline All dairy products & $394(250,695)$ & $442(270,652)^{470}$ \\
\hline Fat ratio $\ddagger$ & $1.4(1.2,1.6)$ & $1.4(1.1,1.6)^{471}$ \\
\hline Fish and fish products & $53(36,77)$ & $39(25,53){ }_{473}^{4 / 2}$ \\
\hline All meat and meat products & $168(114,222)$ & $117(82,162) \quad 474$ \\
\hline Alcohol $\dagger$ & $11(4,19)$ & $3(2,7) \quad 475$ \\
\hline
\end{tabular}

476 NDS, Nordic diet score; E\%, percent of energy intake, mMDS, modified Mediterranean diet 477 score. Values are medians (interquartile ranges).

$478 *$ A ratio of polyunsaturated fatty acids to saturated and trans-fatty acids.

$479 \dagger$ Both diet scores included alcohol, calculated as $100 \%$ ethanol.

$480+$ A ratio of unsaturated fatty acids to saturated fatty acids. 